\title{
THE INITIAL VALUE PROBLEM FOR A DEGENERATE WAVE EQUATION
}

\author{
Y. KANNAI ${ }^{1}$ AND S. KIRO ${ }^{2}$
}

(Communicated by Walter Littman)

\begin{abstract}
The Cauchy problem for a degenerate wave equation, for which the spatial part is essentially a hypoelliptic sum of squares, is solved via a suitable modification of the standard Hilbert space approach for the usual problem.
\end{abstract}

Let $M$ be a compact $C^{\infty}$ manifold and $X_{1}, X_{2}, \ldots, X_{N}$ a family of real vector fields on $M$ satisfying the Hörmander condition.

(H) The Lie algebra over $C^{\infty}(M)$ generated by $X_{1}, X_{2}, \ldots, X_{N}$ is of dimension $\operatorname{dim} M$ at every point of $M$.

The condition (H) implies that the operator $\sum_{j=1}^{N} X_{j}^{2}$ is hypoelliptic with loss of $<2$ derivatives. More generally, the operator

$$
L=\sum_{j=1}^{N}\left(X_{j}^{2}+A_{j} X_{j}\right)+B
$$

where $A_{1}, A_{2}, \ldots, A_{N}, B$ are classical pseudo-differential operators of order 0 , is hypoelliptic with loss of $<2$ derivatives (see [1, Chapter XXII, §2]).

We consider the Cauchy problem for the wave equation of the operator $L$ on $\mathbf{R} \times M$

$$
\begin{gathered}
\left(\frac{\partial}{\partial t}\right)^{2} u(t, x)=L\left(x, D_{x}\right) u(t, x) ; \quad u(t, x)=u_{0}(x), \\
\frac{\partial}{\partial t} u(0, x)=u_{1}(x)
\end{gathered}
$$

or, in the form of a system

$$
\frac{\partial}{\partial t}\left(\begin{array}{l}
u(t, x) \\
v(t, x)
\end{array}\right)=\left(\begin{array}{ll}
0 & I \\
L & 0
\end{array}\right), \quad\left(\begin{array}{l}
u(0, x) \\
v(0, x)
\end{array}\right)=\left(\begin{array}{l}
u_{0}(x) \\
u_{1}(x)
\end{array}\right) .
$$

To define the space of initial data for the problem (1) or (2) we assume, for simplicity, that $M$ carries a nonvanishing $C^{\infty}$-density which will be used to trivialize all density bundles, and, in particular, to define Sobolev spaces $H^{s}(M)(s \in \mathbf{R})$, $H^{0}(M)=L^{2}(M)$. We shall denote by $($,$) and \|\|$ the $L^{2}(M)$-inner product and norm respectively.

Received by the editors July 21,1987 .

1980 Mathematics Subject Classification (1985 Revision). Primary 35L15, 35L80; Secondary $35 \mathrm{H} 05,58 \mathrm{G} 17$.

1 Erica \& Ludwig Jesselson Professor of Theoretical Mathematics.

2 Incumbent of the Lilian and George Lyttle Career Development Chair. 
Following Rothschild and Stein [2] we define the space $S^{\mathbf{1}}(M)$ as the completion of the pre-Hilbert space $C^{\infty}(M)$ equipped with the inner product

$$
\langle f, g\rangle=\sum_{j=1}^{N}\left(X_{j} f, X_{j} g\right)+(f, g) \text {. }
$$

We denote by $\|f\| \|=\langle f, f\rangle^{1 / 2}$ the norm in $S^{1}(M)$.

Rothschild and Stein [2, Theorem 13] had proved the following (the first statement of the assertion is obvious):

LEMMA 1. There is a continuous inclusion $H^{1}(M) \subset S^{1}(M)$ and, if the condition $(\mathrm{H})$ is satisfied, $S^{1}(M) \subset H^{\varepsilon}(M)$ for some $\varepsilon>0$.

The importance of the space $S^{1}(M)$ for the problem (1) is reflected in the following:

LEMMA 2. For $\alpha>0$ small enough, the expression $\operatorname{Re}((I-\alpha L) u, u)^{1 / 2}$ defines a norm on $C^{\infty}(M)$ which is equivalent to the $S^{1}(M)$-norm.

We shall prove Lemma 2 below.

It follows from Lemma 2 that for $\alpha>0$ small enough, the bilinear form $((I-\alpha L) u, v), u, v \in C^{\infty}(M)$ can be extended by continuity to a bounded bilinear form $Q_{\alpha}(u, v)$ on $S^{1}(M)$. Thus

$$
Q_{\alpha}(u, v)=((I-\alpha L) u, v) \quad \text { if } u, v \in C^{\infty}(M) .
$$

Our main result is

THEOREM. For every $u_{0} \in S^{1}(M), u_{1} \in L^{2}(M)$ there is a solution $u(t, x) \in$ $C^{\infty}\left(\mathbf{R}, S^{1}(M)\right)$ of the problem (1) such that for some constants $\alpha, \beta>0$ which do not depend on $u_{0}, u_{1},\left(\operatorname{Re} Q_{\alpha}(u, u)\right)^{1 / 2}$ is a norm on $S^{1}(M)$ which is equivalent to the ||| || norm and

$$
\operatorname{Re} Q_{\alpha}(u, u)+\alpha\left(\frac{\partial u}{\partial t}(t, \cdot), \frac{\partial u}{\partial t}(t, \cdot)\right) \leq e^{\beta t}\left(\operatorname{Re} Q_{\alpha}\left(u_{0}, u_{0}\right)+\alpha\left(u_{1}, u_{1}\right)\right) .
$$

Moreover, if $u_{0}, u_{1} \in C^{\infty}(M)$, then $u(t, x) \in C^{\infty}(\mathbf{R} \times M)$.

For all $s \in \mathbf{R}$, let $\Lambda_{s}$ be an elliptic classical pseudo-differential operator on $M$ establishing an isometry of $L^{2}(M)$ and $H^{s}(M)$ satisfying $\Lambda_{-s} \Lambda_{s}=I$. Note that the theorem can be applied to the operator $\tilde{L}=\Lambda_{-s} L \Lambda_{s}$ since it is of the same form as $L$.

CorollaRy. If $s \in \mathbf{R}, u_{0} \in H^{s+1}(M), u_{1} \in H^{s}(M)$, then $u(t, x)=\Lambda_{s} \tilde{u}(t, x) \in$ $C^{\infty}\left(\mathbf{R}, H^{s+\varepsilon}(M)\right)$ for some $\varepsilon>0$, is a solution of the problem (1), where $\tilde{u}$ is the solution given by the Theorem of the problem

$$
\begin{aligned}
& \left(\frac{\partial}{\partial t}\right)^{2} \tilde{u}(t, x)=\tilde{L} \tilde{u}(t, x), \\
& \tilde{u}(0, x)=\Lambda_{-s} u_{0}(x) \in H^{1}(M) \subset S^{1}(M), \\
& \frac{\partial \tilde{u}}{\partial t}(0, x)=\Lambda_{-s} u_{1}(x) \in L^{2}(M) .
\end{aligned}
$$


Thus the Corollary enables us to find in a standard way the fundamental solution of the Cauchy problem (1) and of the wave equation $(\partial / \partial t)^{2}-L$. We shall prove the Theorem by considering the problem (2) and showing that the operator

$$
\mathscr{L}=\left(\begin{array}{ll}
0, & I \\
L, & 0
\end{array}\right)
$$

is an infinitesimal generator of a certain group. The result is deduced from the properties of this group.

We follow the method of the integration of the Cauchy problem for the usual wave equation due to Yosida (see [3, Chapter XIV, §3]).

We begin the proof by obtaining Lemma 2 . Note that if $X_{j}^{*}$ is the adjoint of $X_{j}$ with respect to the $L^{2}(M)$ inner product, then

$$
X_{j}^{*}=X_{j}+a_{j}
$$

where $a_{j}$ is of order $0, j=1, \ldots, N$.

LEMMA 3. The bilinear form $((I-\alpha L) u, v), u, v \in C^{\infty}(M), \alpha>0$, is continuous with respect to the norm of $S^{1}(M)$.

ProOF. We use (1) and the Schwarz inequality

$$
\begin{aligned}
|((I-\alpha L) u, v)| & \leq\|u\|\|v\|+\alpha \sum_{j=1}^{N}\left(\left|\left(X_{j} u, X_{j}^{*} v\right)\right|+\left|\left(A_{j} X_{j} u, v\right)\right|\right) \\
& \leq\|u\|\|v\|+\alpha \sum_{j=1}^{N}\left(\left\|X_{j} u\right\|\left\|X_{j} v\right\|+\left|\left(X_{j} u, a_{j} v\right)\right|+\left|\left(X_{j} u, A_{j}^{*} v\right)\right|\right) \\
& \leq c_{0}\|u\|\|\mid\| v \|
\end{aligned}
$$

for some constant $c_{0}>0$ which does not depend on $u$ and $v$.

LEMMA 4. There exists a constant $C_{1}>0$ such that for every $u \in C^{\infty}(M)$ and $j=1, \ldots, N$

$$
\left\|X_{j} u\right\|^{2} \leq C_{1}\left(\operatorname{Re}(-L u, u)+\|u\|^{2}\right) .
$$

For the proof of this fact, which can be easily obtained from (4), we refer to [1, Lemma 22.2.2]. Combining Lemma 3 and Lemma 4 we obtain the statement of Lemma 2.

LEMMA 5. For $\alpha>0$ small enough and every $f \in S^{1}(M)$ there exists a unique solution $u \in S^{1}(M)$ of the equation $(I-\alpha L) u=f$.

ProOF. Lemma 2 and the continuity of the extension $Q_{\alpha}(u, v)$ of the bilinear form $((I-\alpha L) u, v)$ imply

$$
\operatorname{Re} Q_{\alpha}(u, u) \geq c \mid\|u\|^{2}
$$

for all $u \in S^{1}(M)$ where $c>0$ does not depend on $u$.

By the Lax-Milgram theorem there exists a bounded linear operator $A: S^{1}(M)$ $\rightarrow S^{1}(M)$ such that

$$
Q_{\alpha}(u, v)=\langle A u, v\rangle, \quad u, v \in S^{1}(M) .
$$


It follows from (5) that

$$
\left\|A u \left|\left\|\left|\left\|v \left|\left\|\geq \operatorname{Re} Q_{\alpha}(u, u) \geq c_{0} \mid\right\| u \|^{2},\right.\right.\right.\right.\right.\right.
$$

hence the range $\mathscr{R}(A)$ of $A$ is closed in $S^{1}(M)$ and there exists a continuous inverse $A^{-1}: \mathscr{R}(A) \rightarrow S^{1}(M)$ of $A$.

If $\mathscr{R}(A)$ is not dense in $S^{1}(M)$ then some $0 \neq h \in S^{1}(M)$ is orthogonal to $\mathscr{R}(A)$.

In particular, $0=\langle h, A h\rangle=Q_{\alpha}(h, h)$ which contradicts (5). Thus $\mathscr{R}(A)=$ $S^{1}(M)$.

Let $u \in S^{1}(M), u_{n} \in C^{\infty}(M), n=1,2, \ldots$, and $u_{n} \rightarrow u$ as $n \rightarrow \infty$ in $S^{1}(M)$. Then $u_{n} \rightarrow u$ in the distribution sense as well and therefore with $(I-\alpha L) u$ considered as a distribution, we have for $g \in C^{\infty}(M)$

$$
(I-\alpha L) u(\bar{g})=\lim _{n \rightarrow \infty}\left((I-\alpha L) u_{n}, g\right)=\lim _{n \rightarrow \infty} Q_{\alpha}\left(u_{n}, g\right)=Q_{\alpha}(u, g)=\langle A u, g\rangle .
$$

Let $f \in S^{1}(M)$. Obviously the conjugate linear functional $\varphi(g)=(f, g)$ is continuous on $S^{1}(M)$. Thus for some $h \in S^{1}(M)$ and $g \in C^{\infty}(M)$,

$$
(f, g)=\langle h, g\rangle=\left\langle A A^{-1} h, g\right\rangle=(I-\alpha L)\left(A^{-1} h\right)(\bar{g}) .
$$

Therefore $f=(I-\alpha L)\left(A^{-1} h\right)$ in the sense of distributions. Uniqueness of the solution is an easy consequence of $(5)$.

Note that by hypoellipticity, if $f \in C^{\infty}(M)$, then the solution $u$ of the equation $(I-\alpha L) u=f$ is also in $C^{\infty}(M)$.

LEMMA 6. If $|n|$ is large enough $f, g \in S^{1}(M)$ then the equation

$$
\left(\mathscr{T}-\frac{1}{n} \mathscr{L}\right)\left(\begin{array}{l}
u \\
v
\end{array}\right)=\left(\begin{array}{l}
f \\
g
\end{array}\right), \quad \mathscr{T}=\left(\begin{array}{ll}
I & 0 \\
0 & I
\end{array}\right),
$$

has a unique solution with $u, v \in S^{1}(M)$ such that for some constants $\alpha, \beta>0$ which do not depend on $f, g$ and $n$, we have $\left(\operatorname{Re} Q_{\alpha}(u, u)\right)^{1 / 2}$ is a norm on $S^{1}(M)$ which is equivalent to the $S^{1}(M)$ norm and

$$
\left(\operatorname{Re} Q_{\alpha}(u, u)+\alpha(v, v)\right)^{1 / 2} \leq\left(1-\frac{\beta}{|n|}\right)^{-1}\left(\operatorname{Re} Q_{\alpha}(f, f)+\alpha(g, g)\right)^{1 / 2} .
$$

Proof. Let $f, g \in S^{1}(M)$. By Lemma 5 there exists for $|n|$ large enough solutions $u_{1}, v_{1} \in S^{1}(M)$ of the equations

$$
\left(I-\frac{1}{n^{2}} L\right) u_{1}=f, \quad\left(I-\frac{1}{n^{2}} L\right) v_{1}=g
$$

Let $u=u_{1}+\frac{1}{n} v_{1}, v=v_{1}+\frac{1}{n} L u_{1}$. Then the pair $\left(\begin{array}{l}u \\ v\end{array}\right)$ is a solution of the equation (6), i.e.

$$
u-\frac{1}{n} v=f, \quad v-\frac{1}{n} L u=g .
$$

Note that if $f, g \in C^{\infty}(M)$ then, by hypoellipticity, $u_{1}, v_{1} \in C^{\infty}(M)$ and hence $u, v \in C^{\infty}(M)$. The uniqueness of the solution and the estimate (7) is obtained 
therefore by continuity from

LEMMA 7. If $u, v \in C^{\infty}(M)$ is the solution of the equation (6) with $f, g \in$ $C^{\infty}(M)$, then the estimate (7) holds.

PROOF. It follows from (8) that

$$
\begin{aligned}
Q_{\alpha}(f, f) & =\left(u-\frac{1}{n} v-\alpha L\left(u-\frac{1}{n} v\right), u-\frac{1}{n} v\right) \\
& =Q_{\alpha}(u, u)-\frac{2}{n} \operatorname{Re}(u, v)+\frac{\alpha}{n}(L v, u)+\frac{\alpha}{n}(L u, v)+\frac{1}{n^{2}} Q_{\alpha}(v, v)
\end{aligned}
$$

and

$$
\begin{aligned}
\alpha(g, g) & =\alpha\left(v-\frac{1}{n} L u, v-\frac{1}{n} L u\right) \\
& =\alpha\left[(v, v)-\frac{1}{n}(L u, v)-\frac{1}{n}(v, L u)+\frac{1}{n^{2}}(L u, L u)\right]
\end{aligned}
$$

Thus

$$
\begin{aligned}
\operatorname{Re} Q_{\alpha}(f, f)+\alpha(g, g) \geq & \operatorname{Re} Q_{\alpha}(u, u)-\frac{2}{n}|\operatorname{Re}(u, v)| \\
& -\frac{\alpha}{|n|}|\operatorname{Re}((v, L u)-(L v, u))|+\alpha(v, v) .
\end{aligned}
$$

It is an easy consequence of Lemma 3 that

$$
|\operatorname{Re}((v, L u)-(L v, u))| \leq C_{1}|\|u|\|\mid\| v \|
$$

for some $C_{1}>0$ which does not depend on $u, v$. Thus

$$
\left.\operatorname{Re} Q_{\alpha}(f, f)+\alpha(g, g) \geq \operatorname{Re} Q_{\alpha}(u, u)+\alpha(v, v)-\frac{1}{|n|}\left(1+\frac{C_{1} \alpha}{2}\right)(\|u \mid\|)^{2}+\|v\|^{2}\right) .
$$

Hence (7) is a consequence of (5) if $\alpha$ is small enough and $|n|$ is large enough.

We consider now the operator $\mathscr{L}$ as defined on the domain $\mathscr{D}(\mathscr{L})=C^{\infty}(M) \times$ $C^{\infty}(M)$ in the Banach space $S^{1}(M) \times L^{2}(M)$ equipped with the norm

$$
\left(\operatorname{Re} Q_{\alpha}(u, u)+\alpha(v, v)\right)^{1 / 2}
$$

Let $\overline{\mathscr{L}}$ be the minimal closed extension of $\mathscr{L}$ with respect to this norm. The estimate (7) implies that the range of the operator $\mathscr{T}-\frac{1}{n} \overline{\mathscr{L}}$ for $|n|$ large enough is closed, and since it contains, by Lemma 6, all elements $(f, g) \in S^{1}(M) \times S^{1}(M)$, it is also dense. Hence, the inverse of the operator $(\mathscr{T}-\mathscr{L} / n)$ for $|n|$ large enough which exists and is continuous by $(7)$, is defined on $S^{1}(M) \times L^{2}(M)$ and

$$
\left\|\left(\mathscr{T}-\frac{1}{n} \mathscr{L}\right)^{-1}\right\| \leq\left(1-\frac{\beta}{|n|}\right)^{-1}
$$

where the operator norm is determined by the norm (9).

The estimate (10) implies that the operator $\overline{\mathscr{L}}$ generates a group $T_{t}$ on $S^{1}(M) \times$ $L^{2}(M)$ (see [3, Chapter X, $\left.\S \S 9,10\right]$ ) such that

$$
\left\|T_{t}\right\| \leq e^{\beta|t|}, \quad t \in \mathbf{R},
$$


$t \rightarrow T_{t}\left(\begin{array}{l}u \\ v\end{array}\right)$ is a $C^{\infty}$-function for $(u, v) \in S^{1}(M) \times L^{2}(M)$, and

$$
\frac{\partial^{k} T_{t}}{\partial t^{k}}\left(\begin{array}{l}
u \\
v
\end{array}\right)=\overline{\mathscr{L}}^{k} T_{t}\left(\begin{array}{l}
u \\
v
\end{array}\right), \quad k=0,1,2, \ldots
$$

(see [3]). Thus

$$
\left(\begin{array}{l}
u(x, t) \\
v(x, t)
\end{array}\right)=T_{t}\left(\begin{array}{l}
u_{0} \\
u_{1}
\end{array}\right)
$$

is a solution of the problem (2) and $u(x, t)$ is a solution of the problem (1) which satisfies the estimate (3) because of (11).

If $u_{0}, u_{1} \in C^{\infty}(M)$ then it is easy to see that because of the estimate we have for the solution $u(t, x)$ defined above

$$
\left(\left(\frac{\partial}{\partial t}\right)^{2}+L\right)^{k} u(t, x) \in L_{\mathrm{loc}}^{2}(\mathbf{R} \times M), \quad k=0,1,2, \ldots
$$

Since the operator $(\partial / \partial t)^{2}+L$ is, by Hörmander's Theorem, hypoelliptic with loss of $<2$ derivatives, it follows that $u(t, x) \in H_{\text {loc }}^{s}(\mathbf{R} \times M)$ for every $s \in \mathbf{R}$, which proves the Theorem.

\section{REFERENCES}

1. L. Hörmander, The analysis of linear partial differential operators. III, Springer-Verlag, Berlin and New York, 1985.

2. L. Rothschild and E. M. Stein, Hypoelliptic differential operators and nilpotent Lie groups, Acta Math. 137 (1976), 246-320.

3. K. Yosida, Functional analysis, 2nd ed., Springer-Verlag, Berlin and New York, 1968.

Department of Theoretical Mathematics, The Weizmann Institute of SCiENCE, REHOVOT 76100, ISRAEL 